\title{
Fuzzy-based Spectrum Handoff in Cognitive Radio Networks
}

\author{
L. Giupponi \\ Centre Tecnològic de Telecomunicacions de Catalunya \\ (CTTC) \\ Av. Canal Olímpic s/n 08860 \\ Castelldefels (Barcelona), Spain \\ lorenza.giupponi@cttc.es
}

\author{
Ana I. Pérez-Neira \\ Universitat Politècnica de Catalunya (UPC) \\ Jordi Girona 1-3 08034 \\ Barcelona, Spain \\ anuska@gps.tsc.upc.edu
}

\begin{abstract}
This paper focuses on spectrum handoffs in a cognitive radio network where secondary (unlisenced) users (i.e. cognitive radios) opportunistically use frequency channels as long as the aggregate interference caused at the primary (licensed) users does not exceed a certain threshold. When harmful interference is caused to a primary user, or when the quality of service perceived by a secondary user is not satisfactory, the secondary user has to initiate a spectrum handoff to quickly vacate the channel it is occupying. The proposal in this paper is a fuzzy-based approach able to make effective spectrum handoff decisions in a context characterized by uncertain, incomplete and heterogeneous information.
\end{abstract}

I. INTRODUCTION

Cognitive Radio is a new paradigm in wireless communications to enhance utilization of the limited spectrum resource. It is defined as a radio able to utilize available side information and to change its transmitter parameters based on the interactions with the surrounding environment where it operates, in order to efficiently use the radio spectrum. The basic idea is that a cognitive radio is able to properly sense the spectrum conditions and, to increase efficiency in spectrum utilization, it seeks to underlay, overlay or interweave its signals with those of the licensed users, without impacting their transmission [1].

In this paper we focus on the underlay paradigm, which encompasses techniques that allow secondary (unlicensed) users (SUs) to communicate provided no significant interference is caused to primary (licensed) users (PUs). In particular, concurrent transmissions of PUs and SUs may occur only if the aggregate interference caused by the SUs at the PUs is maintained below some acceptable threshold. As a result, a hierarchical model is defined where PUs are entities characterized by a higher priority in a given frequency band (e.g. cell phones, TV stations, emergency service, etc.) than SUs. The PUs are not cognitive radio aware, that is, they are not able to facilitate signalling information to SUs to allow the access in their frequencies. On the other hand, SUs are characterized by cognitive radio capabilities, that is, in order to access certain frequencies, a SU has to continually monitor the radio spectrum to identify spectrum opportunities, to reliably detect the presence of PUs, and to evaluate the interference the SUs transmitter may cause on the PUs receivers. This operation is referred to as spectrum sensing. Based on the results collected during spectrum sensing operation, the spectrum has to be managed. The collected information is analyzed and the best available channel is selected by SUs. Additionally, if a PU is detected and if the SU is causing harmful interference to it, the frequency channel has to be quickly vacated initiating the process of the so called spectrum handoff.

Different architectures, both centralized and decentralized, have been proposed in literature for cognitive radio networks. IEEE 802.22 is the first worldwide standard based on the cognitive radio technology [2][3]. In this context a centralized architecture is proposed where a base station manages its own cell and all the associated users. Another example of centralized architecture is DIMSUMnet [4] where a centralized network level brokering mechanism is implemented. On the other hand, since the availability of channels changes over time and space according to the presence of PUs and SUs, distributed control plans in an adhoc fashion have also been proposed to reduce signalling and infrastructure costs of the secondary network. For example, CORVUS [5] is based on local spectrum sensing, where PU detection and spectrum allocation are performed based on the coordinated and collaborative operation of SUs.

In this paper we focus on a decentralized architecture, and we pay particular attention to the problem of the spectrum handoff decision and on the way how a SU makes this decision based on the information available from the spectrum sensing. It is worth mentioning that the same spectrum handoff algorithm proposed in this paper could be applied to a centralized architecture.

The way how SUs make the decision of switching to a different frequency channel, due to the fact that they are causing harmful interference to a PU, is a challenging problem. In fact, a scenario in which PUs and SUs coexist is characterized by high uncertainty deriving from different components. First, the decentralization of the decision procedure makes the decision making inputs regarding the activity of the PUs, their transmission parameters or the position of the PU receivers very vague and imprecise. Second, fading, path loss, interference and noise introduce an intrinsic uncertain component as in any wireless channel. Besides, a major component of disturb derives from the interference introduced by other SUs. Third, the multiple 
decision making inputs (e.g. power levels, signal to noise ratios (SNR), Quality of Service (QoS) indicators, etc.) are heterogeneous and not directly comparable. Finally, the spectrum handoff algorithm implemented at the SU terminal side has to be characterized by low complexity and reduced execution time in order to quickly vacate licensed channels when required.

It is our opinion that the challenges raising in a cognitive radio scenario can be properly met by using techniques based on incomplete knowledge representation and qualitative reasoning. In particular, we propose to use fuzzy logic to deal with the incompleteness, uncertainty and heterogeneity of a cognitive radio scenario, and fuzzy control to implement the decision making process in this kind of scenarios.

Fuzzy logic is a mathematical tool particularly appropriate to make decisions in situations where the available inputs are in general uncertain and imprecise or qualitatively interpreted. Additionally, when the information available about the decision making inputs is mostly heterogeneous, fuzzy logic is able to transform heterogeneous and qualitative information into homogeneous membership values, which can then be processed through a proper set of fuzzy inference rules. As a result, in this paper we propose a fuzzy logic based spectrum handoff algorithm, which can be implemented in a decentralized way, so that SUs can autonomously and automatically make the decision to change its frequency channel. It worth noting that an additional advantage of the proposed approach is its realtime capability, which has been proven many times in practical applications in the framework of fuzzy control. The particular fuzzy logic controller proposed in this paper is simple enough to guarantee low complexity implementation from both software and hardware perspectives and quick spectrum mobility as required in cognitive radio networks, not to harm the detected PUs.

It is worth mentioning that this paper is an initial work and a first contribution to the application of fuzzy logic to cognitive radio networks, consequently its objective is basically to validate the fuzzy option to make decisions in this kind of environments. With this aim, different simplified assumptions will be considered and further works will be required in order to improve the proposed framework and validate it for a more general context. In particular, an important assumption is that the proposed framework is considered for wireless/mobile communications, where PUs are not passive and through their activities (in the form of traffic or signalling information) provide useful side information that the SUs can use to operate in overlay mode. The outline of this paper is organized as follows. In section II, for the reader convenience, we give a brief overview of fuzzy logic and fuzzy logic controllers. In section III, the fuzzy-based spectrum handoff algorithm is presented. Section IV is devoted to the presentation of the simulation scenario and to the discussion of the simulation results. Finally, section V summarizes the conclusion of the paper.

\section{FuZZY LOGIC}

In this section we give a brief introduction of fuzzy logic. The objective is to provide the reader with the basic information necessary for the understanding of the following sections. For a deeper understanding of fuzzy logic, the interested reader is referred to the literature [6][7].
The purpose of fuzzy logic is to realize sophisticated control systems considering that many times real problems cannot be efficiently expressed by means of mathematical models. So, fuzzy set theory models the vagueness that exists in real world problems. According to this theory, when $A$ is a fuzzy set and $x$ is a relevant object, the proposition " $x$ is a member of $A$ " is not necessarily true or false, but it may be true or false only to some degree, the degree to which $x$ is actually a member of $A$. It is common to express degrees of membership in fuzzy sets by numbers on the closed interval $[0,1]$. The extreme values in this interval, 0 and 1 , then represent, respectively, the total denial or affirmation of the membership in the fuzzy set.

In fuzzy logic, each object $x$ can be labelled by a linguistic term, where a linguistic term is a word such as "small", "medium", "large", etc. so that, $x$ is defined as a linguistic variable. Each linguistic variable is associated with a term set $T(x)$, which is the set of names of linguistic values of $x$. Each element in $T(x)$ is a fuzzy set.

A fuzzy set $F$ in a universe of discourse $U$ is characterized by a membership function $\mu_{F}$ which takes values in the interval $[0,1]: \mu_{F}: U \rightarrow[0,1]$.

Let us consider a simple example to clarify these concepts. If "speed" is interpreted as a linguistic variable, then its term set $\mathrm{T}$ (speed) could be defined as: $\mathrm{T}($ speed $)=$ s slow, moderate, fast,... , where each term in $\mathrm{T}$ (speed) is a fuzzy set in the universe of discourse [0,100]. These terms can be characterized as fuzzy sets whose membership functions are shown in Figure 1. We might interpret "slow" as "a speed below about $40 \mathrm{Km} / \mathrm{h}$ ", "moderate" as "a speed close to 55 $\mathrm{Km} / \mathrm{h}$ " and "fast" as "a speed above about $70 \mathrm{Km} / \mathrm{h}$ ".

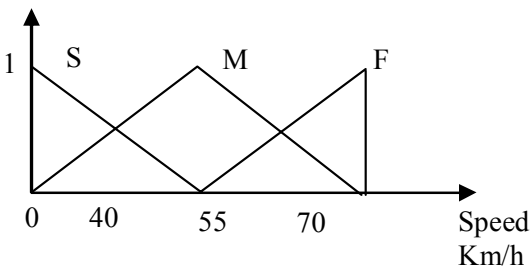

Figure 1 Example of membership function

This is the basic concept of a fuzzy set, which is the generalization of a crisp set, intended to dichotomize the individuals into two groups, i.e. members and nonmembers. In this sense, fuzzy logic provides an inference morphology that enables approximate reasoning capabilities to be applied to knowledge based systems. The theory of fuzzy logic provides a mathematical strength to capture the uncertainties associated with human cognition processes, such as thinking and reasoning.

To implement decision making processes, fuzzy logic makes use of the so called Fuzzy Logic Controllers (FLCs). The essential part of a FLC is a set of linguistic control rules based on expert knowledge in the form:

IF (a set of conditions are satisfied) THEN (a set of consequences can be inferred)

A general FLC consists of four modules: a fuzzy rule base, a fuzzy inference engine and a fuzzification/defuzzification module. A FLC operates by repeating a cycle of five steps implemented by these four modules. First, measurements are taken of all variables that represent relevant conditions of the controlled process. Next, these measurements are converted 
into appropriate fuzzy sets to express measurement uncertainties. This step is called fuzzification. The fuzzified measurements are then used by the inference engine to evaluate control rules stored in the fuzzy rule base. The result of this evaluation is a fuzzy set (or several fuzzy sets) defined on the universe of discourse of possible actions. This fuzzy set is then converted, in the final step of the cycle, into a crisp value (or a vector of values). This conversion is called defuzzification. The defuzzified values represent actions taken by the FLC in individual control cycles.

In essence, the FLC provides an algorithm which can convert the linguistic control strategy based on expert knowledge into an automatic control strategy. In particular, the methodology of FLC appears very useful when the processes are too complex for analysis by conventional quantitative techniques or when the available sources of information are interpreted qualitatively, inexactly or uncertainly, which is the case in a cognitive radio network.

It is worth mentioning that the main weakness of FLCs is the dependability of their decisions on the way how membership functions and fuzzy inference rules are set. To encompass this limit, fuzzy logic is often combined in literature with learning algorithm based on e.g. neural networks or genetic algorithms. This paper, being an initial proposal of the use of fuzzy logic in cognitive radio networks, does not encompass learning approaches to optimize the proposed FLCs, and both the membership functions and the fuzzy rule base are selected based on the expert knowledge [6].

\section{FuZZY-BASEd Spectrum HaNdofF}

The fuzzy-based spectrum handoff proposed in this paper is implemented, without loss of generality, at the SU, so that it is able to make the handoff decision in a decentralized fashion. The most important constraint in a cognitive radio scenario is that of non-interference to the PU receivers. To avoid interference on PU receivers, the SU should have knowledge of its position, which is an extremely challenging research problem [8]-[10]. In this paper we assume that the SUs are able to make estimations about the position of PU receivers. In particular the proposed framework is considered in the context of wireless or mobile communications, where PUs alternatively act as transmitters or receivers, so that a $\mathrm{SU}$, through proper spectrum sensing, is able to detect information about PUs' activity and consequently to approximate their position. Considering the uncertainty which characterizes these estimations, which are affected by the characteristics of the wireless channel, the interference caused by multiple SUs, the decentralization of the decision, etc., they will be realized by means of FLCs.

The proposed algorithm is based on two FLCs, as it is shown in Figure 2. The first one is in charge of estimating the distance between the SU and the PU, and consequently the power at which the $\mathrm{SU}$ should transmit $\left(\mathrm{P}_{\mathrm{SU}}\right)$ without affecting the neighbour PUs. The second FLC, in turn, is in charge of making the spectrum handoff decision. In particular this controller is characterized by two outputs, the first one (HO) indicates whether the handoff has to be realized or not. The second one $\left(\mathrm{MOD}_{\mathrm{PSU}}\right)$ indicates whether the $\mathrm{SU}$ transmission power $\mathrm{P}_{\mathrm{SU}}$ should be modified, in order to avoid a spectrum handoff, so that the spectrum handoff rate can be reduced.
In the following, the FLCs implementing the proposed algorithm and depicted in Figure 2 will be described with more details.

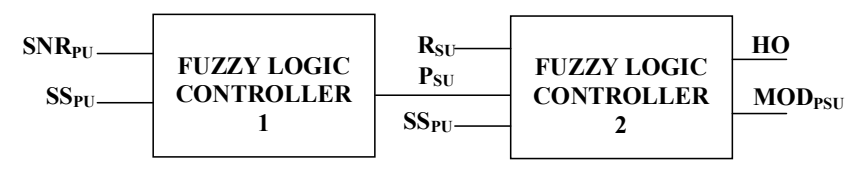

Figure 2 Block scheme of fuzzy-based spectrum handoff algorithm

\section{A. Fuzzy Logic Controller 1}

The first FLC (FLC 1) has been designed to make a first qualitative estimation of the power at which the SU should transmit in order not to affect to the PUs operation. The signal strength received at the $\mathrm{SU}$ from the $\mathrm{PU}\left(\mathrm{SS}_{\mathrm{PU}}\right)$ is not enough information to decide the power at which the SU can transmit. If the signal strength at the SU receiver is "Low" $\left(\mathrm{SS}_{\mathrm{PU}}\right)$, it does not mean that the distance between the PU and the SU is "High", and consequently that the SU transmission power $\left(\mathrm{P}_{\mathrm{SU}}\right)$ can be "High", since the SU has not the information about the power at which the PU is transmitting $\left(\mathrm{P}_{\mathrm{PU}}\right)$. Considering the uncertainty in this scenario, a FLC is proposed (see Figure 3) to qualitatively determine the distance between a SU and a PU, and consequently the transmission power $\mathrm{P}_{\mathrm{SU}}$ at which the $\mathrm{SU}$ should transmit without interfering the PU. We assume that by means of spectral estimation techniques, the $\mathrm{SU}$ is able to qualitatively determine the bit rate of the $\mathrm{PU}\left(\mathrm{R}_{\mathrm{PU}}\right)$. Based on the PU bit rate, an estimation of the signal to noise ratio at the PU $\left(\mathrm{SNR}_{\mathrm{PU}}\right)$ can be deduced [11][12], and consequently also an estimation of the PU transmission power $\left(\mathrm{P}_{\mathrm{PU}}\right)$. As a result, qualitatively comparing the estimation obtained for the PU transmission power $\left(\mathrm{P}_{\mathrm{PU}}\right)$ to the signal strength at the $\mathrm{SU}$ from the PU $\left(\mathrm{SS}_{\mathrm{PU}}\right)$ it is possible to estimate the distance between the PU and the SU. By means of FLC1 each SU can estimate the distance from all the PUs active in its surrounding region. Based on the position of the closer PU, the power $\mathrm{P}_{\mathrm{SU}}$ at which the SU should transmit can be selected.

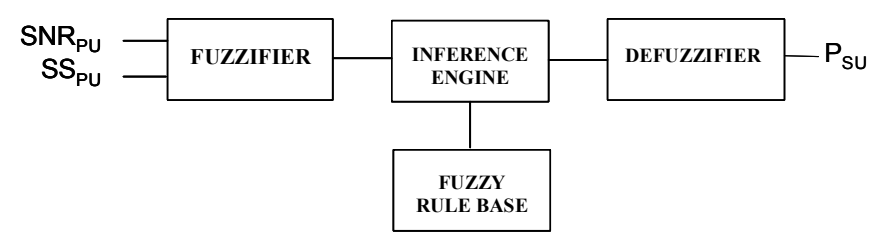

Figure 3 FLC 1 to select the SU transmission power.

The FLC 1 consists of two input linguistic variables (i.e. $\mathrm{SNR}_{\mathrm{PU}}$ and $\mathrm{SS}_{\mathrm{PU}}$ ), characterized by the membership functions depicted in Figure 4, and of one output linguistic variable (i.e. $\mathrm{P}_{\mathrm{SU}}$ ). Each input and output linguistic variable is characterized by a term set of three fuzzy sets, as it is shown in (1).

$T\left(S S_{P U}\right)=T\left(S N R_{P U}\right)=T\left(P_{S U}\right)=\{$ Low, Medium, High $\}$

(1)

Table 1 represents the fuzzy inference rules contained in the FLC 1 fuzzy rule base. The rationale behind their definition is to realize the comparison between the signal strength received by the $\mathrm{SU}$ from the $\mathrm{PU}, \mathrm{SS}_{\mathrm{PU}}$, and the $\mathrm{SNR}_{\mathrm{PU}}$. For example, in rule 3, if the $\mathrm{SNR}_{\mathrm{PU}}$ is "High", but the $\mathrm{SS}_{\mathrm{PU}}$ is "Low", then the distance between PU and SU is estimated to 
be "High", and so can be the $\mathrm{P}_{\mathrm{SU}}$. In turn, if the signal strength received by the SU is "High", the distance between the SU and the PU is considered to be "Low" and consequently the power at which the SU should transmit is also "Low", as it is shown for rules 7, 8 and 9 .

The defuzzification procedure implemented in FLC1 is the centre of area method [6]. The output of this procedure is a value between $0 \mathrm{dBm}$ and the maximum power at which a SU can transmit.
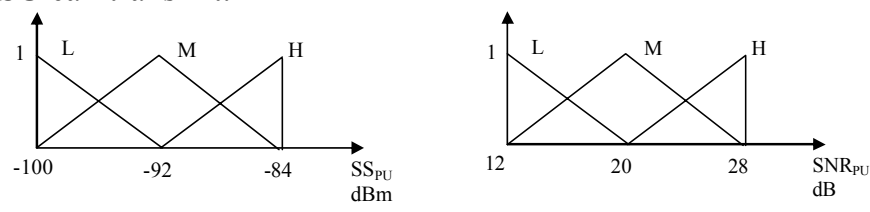

Figure 4 FLC 1Membership functions

Table 1 FLC 1 Fuzzy rule base

\begin{tabular}{|c|c|c|c|}
\hline \multicolumn{3}{|c|}{ IF } & THEN \\
\hline Rule & SS $_{\text {PU }}$ & SNR $_{\text {PU }}$ & P $_{\text {SU }}$ \\
\hline 1 & L & L & L \\
\hline 2 & L & M & M \\
\hline 3 & L & H & H \\
\hline 4 & M & L & L \\
\hline 5 & M & M & L \\
\hline 6 & M & H & M \\
\hline 7 & H & L & L \\
\hline 8 & H & M & L \\
\hline 9 & H & H & L \\
\hline
\end{tabular}

\section{B. Fuzzy Logic Controller 2}

The second FLC (FLC 2) has been designed to qualitatively determine whether a spectrum handoff should be realized or not. In case the SU decides to remain in its frequency channel, its transmission power may be modified in order to reduce the harmful interference on the closer PU. FLC 2 consists of three input linguistic variables, as it is depicted in Figure 2 and Figure 5: 1) the SU transmission power $\mathrm{P}_{\mathrm{SU}} ; 2$ ) the signal strength received at the $\mathrm{SU}, \mathrm{SS}_{\mathrm{PU}} ; 3$ ) the bit rate of the $\mathrm{SU}, \mathrm{R}_{\mathrm{SU}}$, which is considered as an indicator of the quality of service (QoS) perceived by the SU. The corresponding membership functions are represented in Figure 6.

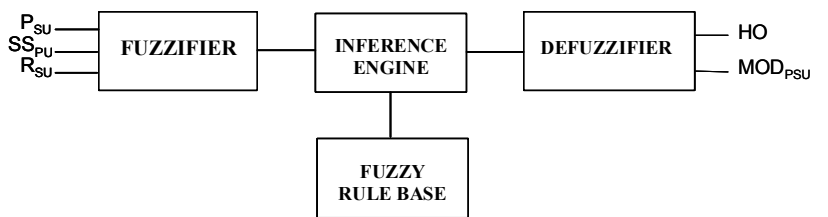

Figure 5 FLC 2 to initiate the spectrum handoff or to modify the $\mathrm{P}_{\mathrm{SU}}$

Each input linguistic variable is characterized by a term set of three fuzzy sets, respectively "Low", "Medium" and "High", as described in (2).

$T\left(P_{S U}\right)=T\left(S S_{P U}\right)=T\left(R_{S U}\right)=\{$ Low, Medium, High $\}$

On the other hand, the FLC 2 consists of two output linguistic variables: $\mathrm{HO}$ and $\mathrm{MOD}_{\mathrm{PSU}} \mathrm{HO}$ decides whether the handoff has to be realized or not, $\mathrm{MOD}_{\mathrm{PSU}}$ indicates whether the SU transmission power should be modified and how. In particular, a spectrum handoff is initiated by a SU if the $\mathrm{HO}<0.5$. Additionally, the $\mathrm{SU}$ transmission power is reduced if $\mathrm{MOD}_{\mathrm{PSU}}<0.5$.

The term sets of these output linguistic variables consist of three fuzzy sets, as it is shown in (3) and (4).

$T\left(M O D_{P S U}\right)=\{$ Low, Medium, High $\}$

$T(H O)=\{$ Yes, Probably Yes, No $\}$

Table 2 contains the FLC2 fuzzy inference rules. A spectrum handoff can be initiated under two circumstances:

1) The QoS of the $S U$ is considered dissatisfactory. An example can be found in rule 4 , where both $\mathrm{P}_{\mathrm{SU}}$ and $\mathrm{R}_{\mathrm{SU}}$ are "Low", so that the SU perception of the service is considered "Low", and the SU decides to initiate a spectrum handoff.

2) The interference caused by the SU on the PU is considered harmful. For example, in rules 25, 26 and 27 the signal strength received by the SU, $\mathrm{SS}_{\mathrm{PU}}$, is "High" and the SU transmission power $\mathrm{P}_{\mathrm{SU}}$ is "High", so that the SU decides to switch to another frequency channel, not to interfere the PU.

Additionally, in order to reduce the spectrum handoff rate, the SU transmission power can be properly modified, through the parameter $\mathrm{MOD}_{\mathrm{PSU}}$, with respect to the first estimation realized through the FLC1. For example, as it is shown in rule 13, if the $\mathrm{P}_{\mathrm{SU}}$ is "Medium" and the $\mathrm{SS}_{\mathrm{PU}}$ is "Medium", instead of switching to a different channel, it is possible to try to reduce the interference caused to the PU by reducing the transmission power of the $\mathrm{SU}, \mathrm{P}_{\mathrm{SU}}$. However, the reduction of the SU transmission power has a negative effect on the SU perception of QoS since the SU signal to interference ratio decreases, so that the rate available at the SU is reduced, as it is also shown by the Shannon relation in (5).

$$
R_{S U}=B_{c} \log \left(1+\frac{P_{S U}}{I+N_{0}}\right)
$$

where $B c$ is the bandwidth of channel c $(\mathrm{Hz}), I$ represents the interference on the $\mathrm{SU}(\mathrm{W})$, and $N_{0}$ is the termal noise.

As a result, even if the interference caused at the PU by the $\mathrm{SU}$ is reduced, the QoS perceived by the SU may be so dissatisfactory that the SU decides to switch to a different channel. This is the case of rule 1 , where the SU initiates a spectrum handoff since both the $\mathrm{P}_{\mathrm{SU}}$ and the $\mathrm{R}_{\mathrm{SU}}$ are "Low".

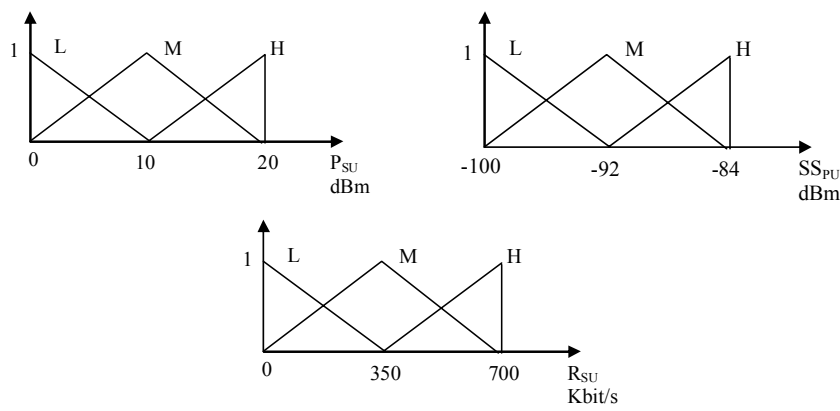

Figure 6 FLC 2 Membership functions 
Table 2 FLC 2 Fuzzy rule base

\begin{tabular}{|c|c|c|c|c|c|}
\hline \multicolumn{5}{|c|}{ IF } & \multicolumn{2}{c|}{ THEN } \\
\hline Rule & $\mathbf{P}_{\text {SU }}$ & SS $_{\text {PU }}$ & R $_{\text {SU }}$ & HO & MOD $_{\text {PSU }}$ \\
\hline 1 & L & L & L & Y & L \\
\hline 2 & L & L & M & PY & L \\
\hline 3 & L & L & H & N & L \\
\hline 4 & L & M & L & Y & L \\
\hline 5 & L & M & M & PY & L \\
\hline 6 & L & M & H & N & L \\
\hline $7,8,9$ & L & H & L, M, H & Y & L \\
\hline $10,11,12$ & M & L & L, M, H & N & M \\
\hline $13,14,15$ & M & M & L, M, H & PY & L \\
\hline $16,17,18$ & M & H & L, M, H & Y & L \\
\hline $19,20,21$ & H & L & L, M, H & N & H \\
\hline $22,23,24$ & H & M & L, M, H & PY & M \\
\hline $25,26,27$ & H & H & L, M, H & Y & M \\
\hline
\end{tabular}

\section{RESUlTS AND DisCUSSION}

In order to evaluate the proposed fuzzy-based spectrum handoff algorithm, a single cell scenario has been considered. A mobility model with users moving according to a random walk model inside the coverage area is adopted with a randomly assigned mobile speed between 0 and 120 $\mathrm{km} / \mathrm{h}$ and a randomly chosen direction. The propagation model considered is given as a function of the distance $d$ by $L(\mathrm{~dB})=128.1+37.6 \log (d(\mathrm{~km}))$. The beginning and the end of the user's activity periods are defined according to a Poisson scheme with an average of 6 calls per hour and user and an average call duration of 180 seconds. The fading is introduced with a standard deviation equal to 7 and the correlation distance is $20 \mathrm{mt}$. The maximum transmission power of both PU and SU is $20 \mathrm{dBm}$. The channel frequency $B c$ is $200 \mathrm{KHz}$.

The algorithm is activated every $100 \mathrm{~ms}$ to identify the need of new spectrum handoffs or new parameter settings. In particular, when the $\mathrm{SU}$ transmission power, $\mathrm{P}_{\mathrm{SU}}$, is modified, it is reduced/increased of $3 \mathrm{~dB}$.

The retained performance measurements are:

Percentage of spectrum handoffs: A spectrum handoff is realized when the FLC 2 makes the corresponding decision.

Interference temperature: The interference temperature is defined as a measure of the RF power available at the PU receiving antenna. This is the power generated by the SUs emitters and by noise sources. More specifically, it is the temperature equivalent of the RF power available at the PU receiving antenna per unit of bandwidth, measured in units of Kelvin [13]. It is calculated as:

$$
T\left(f_{c}, B_{c}\right)=\sum_{i=1}^{N S U} \frac{P_{i}\left(f_{c}, B_{c}\right)}{k B_{c}}
$$

Where $T\left(f_{c}, B_{c}\right)$ is the interference temperature for channel $c$, with central frequency $f c$ and bandwidth $B c$ (in Hertz). $N S U$ is the number of SUs causing harmful interference to the $\mathrm{PU}$ and $P_{i}\left(f_{c}, B_{c}\right)$ is the average interference power in Watts centred at frequency $f c$ and $\mathrm{k}$ is Boltzmann's constant $\left(1.38 \times 10^{-23}\right.$ Joules per Kelvin $)$. According to what envisaged by FCC in [13], SUs can operate in licensed bands in underlay mode, as long as the aggregate interference temperature caused by the SUs at any PU receiver operating in that band does not exceed a predefined threshold. In this paper, we do not focus on this particular threshold and we aim at validating the fuzzy-based approach by showing that it allows decreasing the aggregate interference temperature at the PUs with respect to a reference approach.

The proposed fuzzy-based spectrum handoff algorithm is compared in terms of the described performance indicators to an alternative handoff algorithm based on a fixed strategy. In particular, the $\mathrm{SU}$ transmission power $\mathrm{P}_{\mathrm{SU}}$ is randomly selected and the spectrum handoff is initiated when $\mathrm{SS}_{\mathrm{PU}}>$ $92 \mathrm{dBm}$ or when the $\mathrm{P}_{\mathrm{SU}}<7 \mathrm{dBm}$ or when $\mathrm{R}_{\mathrm{SU}}<350 \mathrm{Kbit} / \mathrm{s}$. The considered scenario is simplified, since in the primary network we consider only one couple of PUs and NSU/2 couples of SUs.

Figure 7 and Figure 8 compare the fuzzy-based approach to the fixed threshold-based approach in terms of percentage of spectrum handoff and interference temperature caused at the PU receiver when $N S U$ is 20 (i.e. 10 couples of SUs are considered). Simulation results depicted in Figure 7 show that the fuzzy-based approach reduces the SU spectrum handoff rate thus guaranteeing a better perception of the service and a reduced signalling cost for the SUs. Additionally, Figure 8 represents the percentage of reduction of interference temperature at the PU receiver when considering the fuzzy-based with respect to the fixed threshold-based approach. All the simulation results are represented as a function of the coverage radius of the primary network where PU and SUs move around. As the radius increases, the spectrum handoff rate is reduced, as well as the interference temperature at the PU, which is reasonable, since the distance between users increases with the coverage radius of the primary network (NSU remains constant). As a result, it has been shown that the fuzzy approach is able to make more intelligent decisions on the most appropriate instant in which initiating the spectrum handoff. Additionally, the opportunity offered by fuzzy logic of linguistically defining the $\mathrm{SU}$ transmission power $\mathrm{P}_{\mathrm{SU}}$ allows to autonomously and automatically adapt it, based on the results of the spectrum sensing operation, in order to control the interference temperature caused to the PU and reduce the spectrum handoff rate.

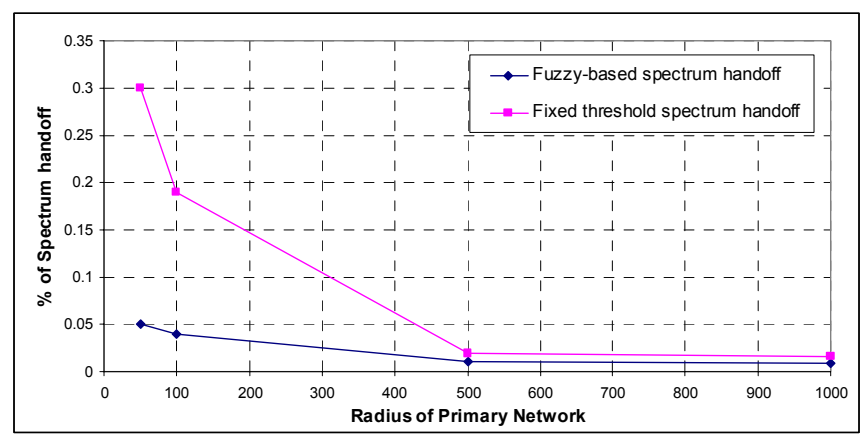

Figure 7 Percentage of spectrum handoffs 


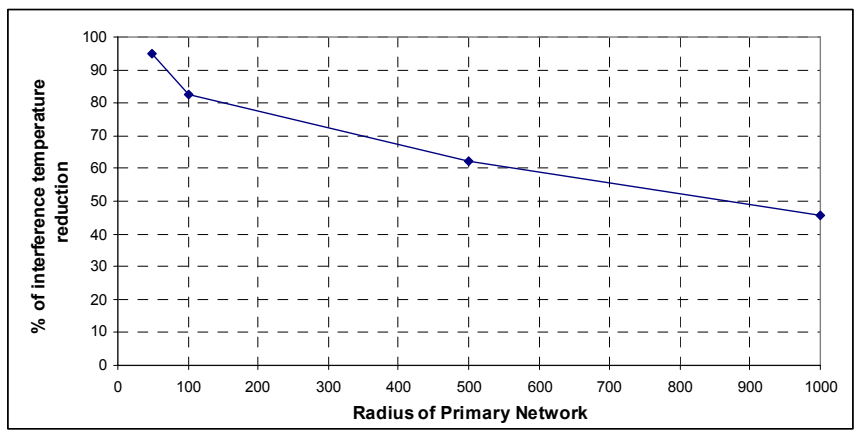

Figure 8 Percentage of reduction of interference temperature at the PU receiver when considering the fuzzy-based approach with respect to the fixed threshold approach

\section{CONCLUSION}

This paper has proposed a solution to initiate spectrum handoffs in a cognitive radio network. It has been shown that the problem of making decisions in a cognitive radio network is extremely challenging. First, the inputs available are confused and heterogeneous due to the characteristics of the wireless channel. Second, the decision making process is not supported by a central entity, but the decisions are made based on incomplete, qualitative and vague information available at the $\mathrm{SU}$ as a result of the spectrum sensing procedure. In this context, the proposed fuzzy-based approach to initiate spectrum handoffs is simple and low computationally complex, which guarantees real-time operation and quick decisions. Simulation results have shown that the fuzzy solution outperforms a solution based on fixed thresholds in terms of spectrum handoff rate and interference temperature measured at the PU receiver.

\section{ACKNOWLEDGEMENT}

This work was supported by the European Commission in the framework of the FP7 Network of Excellence in Wireless COMmunications NEWCOM++ (contract n. 216715).

\section{REFERENCES}

[1]A. Goldsmith, S. A. Jafar, I. Maric, S. Srinivasa, "Breaking Spectrum Gridlock with Cognitive Radios: $\mathrm{Na}$ Information Theoretic Perspective", Proceedings of the IEEE, invited, to appear 2008.

[2]C. Cordeiro, K. Challapali, D. Birru, S. Shankar, IEEE 802.22: the first worldwide wireless standard based on cognitive radios, in Proc. IEEE DySPAN 2005, November 2005, pp. 328-337.

[3] IEEE 802.22 Working group on wireless regional area networks, http://www.ieee802.org/22/

[4] M. M. Buddhikot, P. Kolody, S. Miller, K. Ryan, J. Evans, DIMSUMnet: new directions in wireless networking using coordinated dynamic spectrum access, in: Proc. IEEE WoWMoM 2005, June 2005, pp. 78-85.

[5] D. Cabric, S. M. Mishra, D. Wilkomm, R. Brodersen, A. Wolisz, A Cognitive radio approach for usage of virtual unlisenced spectrum, in: Proc. 14th IST Mobile Wireless Communications Summit, June 2005.

[6] A. Kaufmann, Introduction to Theory of Fuzzy Subsets, New York: Academic, 1975.

[7] H. J. Zimmermann, Fuzzy Sets, Decision Making and Expert Systems, Boston: Kluwer Academic Publisher, 1987.

[8]B. Wild and K. Ramchandran "Detecting primary receivers for cognitive radio applications,", Proceedings of IEEE DySPAN 2005. [9]A. Sahai, R. Tandra, S. Mubaraq, N. Hoven, "Fundamental Design tradeoffs in Cognitive Radio Systems", Proceedings of the first international workshop on Technology and policy for accessing spectrum, Boston, Massachusetts, August 2006.

[10] Niels Hoven and Anant Sahai, "Power Scaling for Cognitive Radio," IEEE International Conf. on Wireless Networks, Communications and Mobile Computing, June 2005.

[11] Part 11: Wireless LAN médium Access control (MAC) and Physical Layer (PHY) specifications. Amendment 4: Further Higher Data Rate Extension in the 2.4 GHz band IEEE Std 802.11g-2003.

[12] Part 11: Wireless LAN médium Access control (MAC) and Physical Layer (PHY) specifications: high-speed physical layer in the $5 \mathrm{GHz}$ band. IEEE 802.11a-1999.

[13] FCC Notice of Inquiry and Notice of Proposed Rule Making, "In the matter of establishment of an interference temperature metric to quantify and manage interference and to expand available unlicensed operation in certain fixed, mobile and satellite frequency bands," ET Docket No. 03-237, November 13, 2003. 